\title{
Space-time discontinuous Galerkin method for the numerical simulation of viscous compressible gas flow with the k-omega turbulence model
}

\author{
Jan $\check{C}^{2} \operatorname{esenek}^{1, \text { a }}$ \\ ${ }^{1}$ VZLU - Czech Aerospace Research Center, Beranových 130, 19905 Praha - Letňany, Czech Republic
}

\begin{abstract}
In this article we deal with the numerical simulation of the non-stationary compressible turbulent flow described by the Reynolds-Averaged Navier-Stokes (RANS) equations. This RANS system is equipped with two-equation k-omega turbulence model. The discretization of these two systems is carried out separately by the space-time discontinuous Galerkin method. This method is based on the piecewise polynomial discontinuous approximation of the sought solution in space and in time. We use the numerical experiments to demonstrate the applicability of the shown approach. All presented results were computed with the own-developed code.
\end{abstract}

\section{Introduction}

During the last decade the space-time discontinuous Galerkin finite element method (ST-DG), which is based on piecewise polynomial discontinuous approximations of the sought solution, became very popular in the field of numerical simulation of the fluid flow. This method of higher order was successfully used for the simulation of the NavierStokes equations [1-6]. In the case of compressible turbulent flow the finite volume - space-time discontinuous Galerkin method was used [7-9], where the equations of the turbulence model were discretized by the implicit finite volume method. This article is devoted to the discretization of viscous compressible turbulent gas flow using STDG applied also for the equations of the turbulence model. The flow is described by the system of the RANS equations equipped with the system of $k-\omega$ equations. These two systems are solved separately and discretized by STDG. In this article Wilcox $k-\omega$ turbulence model was chosen [10]. The flow around airfoil RAE2822 will be used for the numerical simulations. These results are compared with experiments. Computational results show that the STDG method is good approach for the solution of these types of problems.

\section{Formulation of the $k-\omega$ turbulence model}

We consider compressible turbulent flow in a bounded domain $\Omega \subset R^{2}$. We assume that the boundary $\Omega$ consists of three disjoint parts $\partial \Omega=\Gamma_{I} \cup \Gamma_{O} \cup \Gamma_{W}$, where $\Gamma_{I}$ is the inlet, $\Gamma_{O}$ is the outlet and $\Gamma_{W}$ is impermeable wall.

The system of the RANS equations describing the viscous compressible turbulent flow can be written in the form

$$
\frac{\partial \mathbf{w}}{\partial t}+\sum_{s=1}^{2} \frac{\partial \mathbf{f}_{s}(\mathbf{w})}{\partial x_{s}}+\sum_{s=1}^{2} \frac{\partial \mathbf{f}_{s}^{p}(\mathbf{w})}{\partial x_{s}}
$$

a e-mail: jan.cessa@seznam.cz, cesenek@vzlu.cz

$$
=\sum_{s=1}^{2} \frac{\partial \mathbf{k}_{s}(\mathbf{w})}{\partial x_{s}}+\sum_{s=1}^{2} \frac{\partial \mathbf{R}_{s}(\mathbf{w}, \nabla \mathbf{w})}{\partial x_{s}}
$$

where for $s=1,2$ we have

$$
\begin{aligned}
\mathbf{w}= & \left(w_{1}, \ldots, w_{4}\right)^{T}=\left(\rho, \rho v_{1}, \rho v_{2}, E\right)^{T} \in R^{4}, \\
\mathbf{f}_{s}(\mathbf{w})= & \left(f_{s, 1}, \ldots, f_{s, 4}\right)^{T} \\
= & \left(\rho v_{s}, \rho v_{1} v_{s}+\delta_{1 s} p,\right. \\
& \left.\rho v_{2} v_{s}+\delta_{2 s} p,(E+p) v_{s}\right)^{T}, \\
\mathbf{f}_{s}^{p}(\mathbf{w})= & \left(f_{s, 1}^{p}, \ldots, f_{s, 4}^{p}\right)^{T} \\
= & \left(0, \frac{2}{3} \rho k \delta_{1 s}, \frac{2}{3} \rho k \delta_{2 s}, \frac{2}{3} \rho k v_{s}\right)^{T}, \\
\mathbf{R}_{s}(\mathbf{w}, \nabla \mathbf{w})= & \left(R_{s, 1}, \ldots, R_{s, 4}\right)^{T} \\
& =\left(0, \tau_{s 1}^{V}, \tau_{s 2}^{V}, \sum_{r=1}^{2} \tau_{s r}^{V} v_{r}+\left(\frac{c_{p} \mu_{L}}{P r}+\frac{c_{p} \mu_{T}}{P r_{T}}\right) \frac{\partial \theta}{\partial x_{s}}\right)^{T}, \\
\mathbf{k}_{s}(\mathbf{w})= & \left(k_{s, 1}, \ldots, k_{s, 4}\right)^{T} \\
= & \left(0, \ldots, 0,\left(\mu_{L}+\sigma_{k} \frac{\rho k}{e^{\tilde{\omega}}}\right) \frac{\partial k}{\partial x_{s}}\right)^{T},
\end{aligned}
$$

where

$$
\begin{aligned}
\tau_{s r}^{V} & =-\frac{2}{3}\left(\mu_{L}+\mu_{T}\right) \operatorname{div} \boldsymbol{v} \delta_{s r}+2\left(\mu_{L}+\mu_{T}\right) d_{s r}(\boldsymbol{v}), \\
d_{s r}(\boldsymbol{v}) & =\frac{1}{2}\left(\frac{\partial v_{s}}{\partial x_{r}}+\frac{\partial v_{r}}{\partial x_{s}}\right) .
\end{aligned}
$$

We use the following notation: $\boldsymbol{v}=\left(v_{1}, v_{2}\right)$ - velocity, $\rho$ - density, $p$ - pressure, $\theta$ - absolute temperature, $E$ - total energy, $\gamma$ - Poisson adiabatic constant, $\kappa$ - heat conduction coefficient, $c_{v}$ - specific heat at constant volume, $c_{p}$ - specific heat at constant pressure, where $c_{p}=\gamma c_{v}, \operatorname{Pr}$ is the laminar Prandtl number, which can be express in the form $\operatorname{Pr}=\frac{c_{p} \mu_{L}}{\kappa}, \operatorname{Pr}_{T}$ is the turbulent Prandtl constant number, $\mu_{T}$ is the eddy-viscosity coefficient, $\mu_{L}$ is the dynamic viscosity coefficient dependent on temperature via Sutherland's 
formula. The above system is completed by the thermodynamical relations

$$
p=(\gamma-1)\left(E-\frac{1}{2} \rho|\boldsymbol{v}|^{2}-\rho k\right), \theta=\frac{1}{c_{v}}\left(\frac{E}{\rho}-\frac{1}{2}|\boldsymbol{v}|^{2}-k\right)
$$

and is equipped with the initial condition

$$
\mathbf{w}(x, 0)=\mathbf{w}^{0}(x), \quad x \in \Omega
$$

and the following boundary conditions

a) $\left.\rho\right|_{\Gamma_{I}}=\rho_{D}$,

b) $\boldsymbol{v}_{\Gamma_{I}}=\boldsymbol{v}_{D}=\left(v_{D 1}, v_{D 2}\right)^{T}$,

c) $\sum_{s, r=1}^{2} \tau_{s r}^{V} n_{s} v_{r}+\left(\frac{c_{p} \mu_{L}}{P r}+\frac{c_{p} \mu_{T}}{P r_{T}}\right) \frac{\partial \theta}{\partial n}=0 \quad$ on $\Gamma_{I}$,

a) $\left.\boldsymbol{v}\right|_{\Gamma_{W}}=0$,

b) $\left.\frac{\partial \theta}{\partial n}\right|_{\Gamma_{W}}=0$

a) $\sum_{s=1}^{2} \tau_{s r}^{V} n_{s}=0, r=1,2 \quad$ on $\Gamma_{O}, \quad$ b) $\left.\frac{\partial \theta}{\partial n}\right|_{\Gamma_{O}}=0$,

with given data $\mathbf{w}^{0}, \rho_{D}, \boldsymbol{v}_{D}$. It is possible to show that $\mathbf{f}_{s}(\alpha \mathbf{w})=$ $\alpha \mathbf{f}_{s}(\mathbf{w})$ for $\alpha>0$. This property implies that

$$
\mathbf{f}_{s}(\mathbf{w})=\mathbf{A}_{s}(\mathbf{w}) \mathbf{w}, \quad s=1,2,
$$

where

$$
\mathbf{A}_{s}(\mathbf{w})=\frac{D \mathbf{f}_{s}(\mathbf{w})}{D \mathbf{w}}, \quad s=1,2,
$$

are the Jacobi matrices of the mappings $\mathbf{f}_{s}$. Similary we can express

$$
\mathbf{f}_{s}^{p}(\mathbf{w})=\mathbf{A}_{s}^{p}(\mathbf{w}) \mathbf{w}, \quad s=1,2 .
$$

The viscous terms $\mathbf{R}_{s}(\mathbf{w}, \nabla \mathbf{w})$ can be expressed in the form

$$
\mathbf{R}_{s}(\mathbf{w}, \nabla \mathbf{w})=\sum_{k=1}^{2} \mathbf{K}_{s, k}(\mathbf{w}) \frac{\partial \mathbf{w}}{\partial x_{k}}, \quad s=1,2,
$$

where $\mathbf{K}_{s, k}(\mathbf{w}) \in R^{4 \times 4}$ are matrices depending on $\mathbf{w}$.

The aforesaid system of the RANS equations is completed by the Wilcox's turbulence model (see [10]) for $\mu_{T}$. For the sake of the stability we introduce new variable $\tilde{\omega}=\ln \omega$. (For details see [11]). Then the turbulence model reads

$$
\frac{\partial \rho \tilde{\mathbf{w}}}{\partial t}+\sum_{s=1}^{2} \frac{\partial \tilde{\mathbf{f}}_{s}(\tilde{\mathbf{w}})}{\partial x_{s}}=\sum_{s=1}^{2} \frac{\partial \tilde{\mathbf{R}}_{s}(\tilde{\mathbf{w}}, \nabla \tilde{\mathbf{w}})}{\partial x_{s}}+\tilde{\mathbf{s}}(\tilde{\mathbf{w}}),
$$

where for $s=1,2$ we have

$$
\begin{aligned}
\tilde{\mathbf{w}}= & \left(\tilde{w}_{1}, \tilde{w}_{2}\right)^{T}=(\tilde{\omega}, k)^{T} \in R^{2}, \\
\tilde{\mathbf{f}}_{s}(\tilde{\mathbf{w}})= & \left(\tilde{f}_{s, 1}, \tilde{f}_{s, 2}\right)^{T}=\left(\rho \tilde{\omega} v_{s}, \rho k v_{s}\right)^{T}, \\
\tilde{\mathbf{R}}_{s}(\tilde{\mathbf{w}}, \nabla \tilde{\mathbf{w}})= & \left(\tilde{R}_{s, 1}, \tilde{R}_{s, 2}\right)^{T}= \\
& \left(\left(\mu_{L}+\sigma_{\omega} \mu_{T}\right) \frac{\partial \tilde{\omega}}{\partial x_{s}},\left(\mu_{L}+\sigma_{k} \mu_{T}\right) \frac{\partial k}{\partial x_{s}}\right)^{T}, \\
\tilde{\mathbf{s}}(\tilde{\mathbf{w}})= & \left(\alpha_{\omega} \frac{P_{k}}{k}-\beta \rho e^{\tilde{\omega}}+\sum_{s=1}^{2}\left(\mu_{L}+\sigma_{\omega} \mu_{T}\right) \frac{\partial \tilde{\omega}}{\partial x_{s}} \frac{\partial \tilde{\omega}}{\partial x_{s}}+\tilde{C}_{D},\right. \\
& \left.P_{k}-\beta^{*} \rho e^{\tilde{\omega}} k\right)^{T} .
\end{aligned}
$$

Here $\omega$ is the turbulence dissipation, $k$ is the turbulence kinetic energy and $\mu_{T}$ is the eddy viscosity. We can write the production term as

$$
P_{k}=\sum_{s=1}^{2} \sum_{r=1}^{2} \tau_{s r}^{T} \frac{\partial v_{s}}{\partial x_{r}}
$$

where

$$
\begin{aligned}
\tau_{s r}^{T} & =-\frac{2}{3} \mu_{T} \operatorname{div} \boldsymbol{v} \delta_{s r}-\frac{2}{3} \rho k \delta_{s r}+2 \mu_{T} d_{s r}(\boldsymbol{v}), \\
d_{s r}(\boldsymbol{v}) & =\frac{1}{2}\left(\frac{\partial v_{s}}{\partial x_{r}}+\frac{\partial v_{r}}{\partial x_{s}}\right), \\
\mu_{T} & =\frac{\rho k}{\omega_{l i m}} .
\end{aligned}
$$

Limited eddy viscosity $\omega_{\text {lim }}$ is given by the term

$$
\begin{aligned}
& \omega_{l i m}=\max \left\{e^{\tilde{\omega}}, C_{l i m} \sqrt{\left.\frac{1}{2 \beta^{*}} \sum_{r, s=1}^{2} \tilde{\tau}_{r s} \tilde{\tau}_{r s}\right\}},\right. \\
& \tilde{\tau}_{r s}=-\frac{2}{3} \operatorname{div} \boldsymbol{v} \delta_{r s}+2 d_{r s}(\boldsymbol{v}) .
\end{aligned}
$$

The cross-diffusion term $C_{D}$ is defined as

$$
C_{D}=\sigma_{D} \frac{\rho}{e^{\tilde{\omega}}} \max \left\{\sum_{s=1}^{2} \frac{\partial k}{\partial x_{s}} \frac{\partial \tilde{\omega}}{\partial x_{s}}, 0\right\}
$$

The coefficients $\beta, \beta^{*}, \sigma_{k}, \sigma_{\omega}, \sigma_{D}, \alpha_{\omega}, C_{\text {lim }}, \operatorname{Pr}_{T}$ are chosen by [10]:

$$
\begin{aligned}
& \alpha_{\omega}=\frac{13}{25}, \quad \beta=0.0708, \quad \beta^{*}=0.09, \quad \sigma_{k}=0.6, \quad \sigma_{\omega}=0.5 \\
& C_{l i m}=\frac{7}{8}, \quad \sigma_{D}=\frac{1}{8}, \quad \operatorname{Pr}_{T}=\frac{8}{9} .
\end{aligned}
$$

This system is also equipped with the initial condition

$$
\tilde{\mathbf{w}}(x, 0)=\tilde{\mathbf{w}}^{0}(x), \quad x \in \Omega
$$

and the following boundary conditions
a) $\left.\tilde{\omega}\right|_{\Gamma_{I}}=\tilde{\omega}_{D}$
b) $\left.k\right|_{\Gamma_{I}}=k_{D}$,
a) $\left.\tilde{\omega}\right|_{\Gamma_{W}}=\tilde{\omega}_{\text {wall }}$
b) $\left.k\right|_{\Gamma_{W}}=0$,
a) $\left.\frac{\partial \tilde{\omega}}{\partial n}\right|_{\Gamma_{O}}=0$
b) $\left.\frac{\partial k}{\partial n}\right|_{\Gamma_{O}}=0$,

with given data $\tilde{\mathbf{w}}^{0}, \tilde{\omega}_{D}, k_{D}, \tilde{\omega}_{\text {wall }}$. Similary like in the RANS case we can express convect terms

$$
\tilde{\mathbf{f}}_{s}^{p}(\mathbf{w})=\tilde{\mathbf{A}}_{s}^{p}(\tilde{\mathbf{w}}) \tilde{\mathbf{w}}, \quad s=1,2
$$

and diffusion terms

$$
\tilde{\mathbf{R}}_{s}(\tilde{\mathbf{w}}, \nabla \tilde{\mathbf{w}})=\tilde{\mathbf{K}}_{s}(\tilde{\mathbf{w}}) \frac{\partial \tilde{\mathbf{w}}}{\partial x_{s}}, \quad s=1,2,
$$

where $\tilde{\mathbf{K}}_{s}(\mathbf{w}) \in R^{2 \times 2}$ are matrices depending on $\tilde{\mathbf{w}}$. 


\section{Discretization}

\subsection{Space discretization of the problem}

By $\Omega_{h}$ we denote polygonal approximation of the domain $\Omega$. Let $\mathcal{T}_{h}$ be a partition of the domain $\Omega_{h}$ into finite number of closed elements with mutually disjoint interiors such that $\overline{\Omega_{h}}=\bigcup_{K \in \mathcal{T}_{h}} K$. In 2D problems, we usually choose $K \in \mathcal{T}_{h}$ as triangles or quadrilaterals. By $\mathcal{F}_{h}$ we denote the system of all faces of all elements $K \in \mathcal{T}_{h}$. Further, we introduce the set of boundary faces $\mathcal{F}_{h}^{B}=\left\{\Gamma \in \mathcal{F}_{h} ; \Gamma \subset \partial \Omega_{h}\right\}$ and the set of inner faces $\mathcal{F}_{h}^{I}=\mathcal{F}_{h} \backslash \mathcal{F}_{h}^{B}$. Each $\Gamma \in \mathcal{F}_{h}$ is associated with a unit normal vector $\boldsymbol{n}_{\Gamma}$. For $\Gamma \in \mathcal{F}_{h}^{B}$ the normal $\boldsymbol{n}_{\Gamma}$ has the same orientation as the outer normal to $\partial \Omega_{h}$. For each $\Gamma \in \mathcal{F}_{h}^{I}$ there exist two neighbouring elements $K_{\Gamma}^{L}, K_{\Gamma}^{R} \in \mathcal{T}_{h}$ such that $\Gamma \subset \partial K_{\Gamma}^{L} \cap \partial K_{\Gamma}^{R}$. We use the convention that $K_{\Gamma}^{R}$ lies in the direction of $\boldsymbol{n}_{\Gamma}$ and $K_{\Gamma}^{L}$ lies in the opposite direction to $\boldsymbol{n}_{\Gamma}$. If $\Gamma \in \mathcal{F}_{h}^{B}$, then the element adjacent to $\Gamma$ will be denoted by $K_{\Gamma}^{L}$.

We shall look for an approximate solution of the problem in the space of piecewise polynomial functions

$$
\mathbf{S}_{h}^{p}=\left(S_{h}^{p}\right)^{4}, \quad S_{h}^{p}=\left\{v ;\left.v\right|_{K} \in P^{p}(K), \forall K \in \mathcal{T}_{h}\right\},
$$

where $p>0$ is an integer and $P^{p}(K)$ denotes the space of all polynomials on $K$ of degree $\leq p$. A function $\Phi \in \mathbf{S}_{h}^{p}$ is, in general, discontinuous on interfaces $\Gamma \in \mathcal{F}_{h}^{I}$. By $\Phi_{\Gamma}^{L}$ and $\Phi_{\Gamma}^{R}$ we denote the values of $\Phi$ on $\Gamma$ considered from the interior and the exterior of $K_{\Gamma}^{L}$, respectively, and set

$$
\begin{aligned}
& \langle\Phi\rangle_{\Gamma}=\frac{1}{2}\left(\Phi_{\Gamma}^{L}+\Phi_{\Gamma}^{R}\right), \\
& {[\Phi]_{\Gamma}=\Phi_{\Gamma}^{L}-\Phi_{\Gamma}^{R},}
\end{aligned}
$$

which denotes the average and jump of $\Phi$ on $\Gamma$.

The discrete problem is derived in the following way: For arbitrary $t \in[0, T]$ we can multiply the system by a test function $\mathbf{S}_{h}^{p}$, integrate over $K \in \mathcal{T}_{h}$, apply Green's theorem, sum over all elements $K \in \mathcal{T}_{h}$, use the concept of the numerical flux and introduce suitable terms mutually vanishing for a regular exact solution. Moreover, we carry out a suitable partial linearization of nonlinear terms and then we can define the following forms.

In order to evaluate the integrals over $\Gamma \in \mathcal{F}_{h}$ in inviscid term we use the approximation

$$
\mathbf{H}\left(\mathbf{w}_{\Gamma}^{L}, \mathbf{w}_{\Gamma}^{R}, \boldsymbol{n}_{\Gamma}\right) \approx \sum_{s=1}^{2} \mathbf{f}_{s}(\mathbf{w})\left(\boldsymbol{n}_{\Gamma}\right)_{s}
$$

where $\mathbf{H}$ is a numerical flux. For the construction of the numerical flux we use the properties (2) of $\mathbf{f}_{s}$. Let us define the matrix

$$
\mathbf{P}(\mathbf{w}, \boldsymbol{n}):=\sum_{s=1}^{2} \mathbf{A}_{s}(\mathbf{w}) n_{s}
$$

where $\boldsymbol{n}=\left(n_{1}, n_{2}\right), n_{1}^{2}+n_{2}^{2}=1$. Then we have

$$
\mathbf{P}(\mathbf{w}, \boldsymbol{n}) \mathbf{w}:=\sum_{s=1}^{2} \mathbf{f}_{s}(\mathbf{w}) n_{s} .
$$

It is possible to show that the matrix $\mathbf{P}$ is diagonalizable. It means that there exists a nonsingular matrix $\mathbf{T}=\mathbf{T}(\mathbf{w}, \boldsymbol{n})$ and a diagonal matrix $\Lambda=\Lambda(\mathbf{w}, \boldsymbol{n})$ such that

$$
\mathbf{P}=\mathbf{T} \Lambda \mathbf{T}^{-1}, \quad \Lambda=\operatorname{diag}\left(\lambda_{1}, \ldots, \lambda_{4}\right),
$$

where $\lambda_{i}=\lambda_{i}(\mathbf{w}, \boldsymbol{n})$ are eigenvalues of the matrix $\mathbf{P}$. Then we can define the "positive" and "negative" parts of the matrix $\mathbf{P}$ by

$$
\mathbf{P}^{ \pm}=\mathbf{T} \Lambda^{ \pm} \mathbf{T}^{-1}, \quad \Lambda^{ \pm}=\operatorname{diag}\left(\lambda_{1}^{ \pm}, \ldots, \lambda_{4}^{ \pm}\right),
$$

where $\lambda^{+}=\max (\lambda, 0), \lambda^{-}=\min (\lambda, 0)$. Using this concept, we introduce the so-called Vijayasundaram numerical flux

$$
\mathbf{H}\left(\mathbf{w}^{L}, \mathbf{w}^{R}, \boldsymbol{n}\right)=\mathbf{P}^{+}\left(\frac{\mathbf{w}^{L}+\mathbf{w}^{R}}{2}, \boldsymbol{n}\right) \mathbf{w}^{L}+\mathbf{P}^{-}\left(\frac{\mathbf{w}^{L}+\mathbf{w}^{R}}{2}, \boldsymbol{n}\right) \mathbf{w}^{R} .
$$

This numerical flux has suitable form for a linearization. Now we can define inviscid form in the following way

$$
\begin{aligned}
& b_{h}\left(\overline{\mathbf{w}}_{h}, \mathbf{w}_{h}, \Phi_{h}\right):= \\
& -\sum_{K \in \mathcal{T}_{h}} \int_{K} \sum_{s=1}^{2} \mathbf{A}_{s}\left(\overline{\mathbf{w}}_{h}\right) \mathbf{w}_{h} \cdot \frac{\partial \Phi_{h}}{\partial x_{s}} \mathrm{~d} \boldsymbol{x} \\
& +\sum_{\Gamma \in \mathcal{F}_{h}^{I}} \int_{\Gamma}\left(\mathbf{P}^{+}\left(\left\langle\overline{\mathbf{w}}_{h}\right\rangle, \boldsymbol{n}_{\Gamma}\right) \mathbf{w}_{h}^{L}+\mathbf{P}^{-}\left(\left\langle\overline{\mathbf{w}}_{h}\right\rangle, \boldsymbol{n}_{\Gamma}\right) \mathbf{w}_{h}^{R}\right) \cdot\left[\Phi_{h}\right] \mathrm{d} S \\
& +\sum_{\Gamma \in \mathcal{F}_{h}^{B}} \int_{\Gamma}\left(\mathbf{P}^{+}\left(\left\langle\overline{\mathbf{w}}_{h}\right\rangle, \boldsymbol{n}_{\Gamma}\right) \mathbf{w}_{h}^{L}+\mathbf{P}^{-}\left(\left\langle\overline{\mathbf{w}}_{h}\right\rangle, \boldsymbol{n}_{\Gamma}\right) \overline{\mathbf{w}}_{h}^{R}\right) \cdot \Phi_{h} \mathrm{~d} S,
\end{aligned}
$$

where the boundary state $\overline{\mathbf{w}}_{h}^{R}$ is evaluated with the aid of the local linearized Riemann problem described in [1]. For the discretization of the viscous terms we use the property (3) and get the viscous form

$$
\begin{aligned}
& a_{h}\left(\overline{\mathbf{w}}_{h}, \mathbf{w}_{h}, \Phi_{h}\right):= \\
& +\sum_{K \in \mathcal{T}_{h}} \int_{K} \sum_{s=1}^{2} \sum_{k=1}^{2} \mathbf{K}_{s, k}\left(\overline{\mathbf{w}}_{h}\right) \frac{\partial \mathbf{w}_{h}}{\partial x_{k}} \cdot \frac{\partial \Phi_{h}}{\partial x_{s}} \mathrm{~d} \boldsymbol{x} \\
& \left.-\sum_{\Gamma \in \mathcal{F}_{h}^{I}} \int_{\Gamma} \sum_{s=1}^{2} / \sum_{k=1}^{2} \mathbf{K}_{s, k}\left(\overline{\mathbf{w}}_{h}\right) \frac{\partial \mathbf{w}_{h}}{\partial x_{k}}\right\rangle\left(\boldsymbol{n}_{\Gamma}\right)_{s} \cdot\left[\Phi_{h}\right] \mathrm{d} S \\
& -\sum_{\Gamma \in \mathcal{F}_{h}^{B}} \int_{\Gamma} \sum_{s=1}^{2} \sum_{k=1}^{2} \mathbf{K}_{s, k}\left(\overline{\mathbf{w}}_{h}\right) \frac{\partial \mathbf{w}_{h}}{\partial x_{k}}\left(\boldsymbol{n}_{\Gamma}\right)_{s} \cdot \Phi_{h} \mathrm{~d} S \\
& -\Theta \sum_{\Gamma \in \mathcal{F}_{h}^{I}} \int_{\Gamma} \sum_{s=1}^{2}\left\langle\sum_{k=1}^{2} \mathbf{K}_{k, s}^{T}\left(\overline{\mathbf{w}}_{h}\right) \frac{\partial \Phi_{h}}{\partial x_{k}}\right\rangle\left(\boldsymbol{n}_{\Gamma}\right)_{s} \cdot\left[\mathbf{w}_{h}\right] \mathrm{d} S \\
& -\Theta \sum_{\Gamma \in \mathcal{F}_{h}^{B}} \int_{\Gamma} \sum_{s=1}^{2} \sum_{k=1}^{2} \mathbf{K}_{k, s}^{T}\left(\overline{\mathbf{w}}_{h}\right) \frac{\partial \Phi_{h}}{\partial x_{k}}\left(\boldsymbol{n}_{\Gamma}\right)_{s} \cdot \mathbf{w}_{h} \mathrm{~d} S
\end{aligned}
$$

We set $\Theta=1$ or $\Theta=0$ or $\Theta=-1$ and get the so-called symmetric version (SIPG) or incomplete version (IIPG) or nonsymetric version (NIPG), respectively, of the discretization of the viscous terms.

Further, we define the turbulent forms $p_{h}, k_{h}$, the interior and boundary penalty form $J_{h}^{\sigma}$ and the right-hand side form $l_{h}$ in the following way:

$$
p_{h}\left(\overline{\mathbf{w}}_{h}, \mathbf{w}_{h}, \Phi_{h}\right):=\sum_{\Gamma \in \mathcal{F}_{h}^{I}} \int_{\Gamma} \sum_{s=1}^{2} \mathbf{A}_{s}^{p}\left(\overline{\mathbf{w}}_{h}^{L}\right) \mathbf{w}_{h}^{L} \cdot \Phi_{h}^{L}\left(\boldsymbol{n}_{\Gamma}\right)_{s} \mathrm{~d} S
$$




$$
\begin{aligned}
& -\sum_{\Gamma \in \mathcal{F}_{h}^{I}} \int_{\Gamma} \sum_{s=1}^{2} \mathbf{A}_{s}^{p}\left(\overline{\mathbf{w}}_{h}^{R}\right) \mathbf{w}_{h}^{R} \cdot \Phi_{h}^{R}\left(\boldsymbol{n}_{\Gamma}\right)_{s} \mathrm{~d} S \\
& +\sum_{\Gamma \in \mathcal{F}_{h}^{B}} \int_{\Gamma} \sum_{s=1}^{2} \mathbf{A}_{s}^{p}\left(\overline{\mathbf{w}}_{h}\right) \mathbf{w}_{h} \cdot \Phi_{h}\left(\boldsymbol{n}_{\Gamma}\right)_{s} \mathrm{~d} S \\
& -\sum_{K \in \mathcal{T}_{h}} \int_{K} \sum_{s=1}^{2} \mathbf{A}_{s}^{p}\left(\overline{\mathbf{w}}_{h}\right) \mathbf{w}_{h} \cdot \frac{\partial \Phi_{h}}{\partial x_{s}} \mathrm{~d} \boldsymbol{x}, \\
& k_{h}\left(\overline{\mathbf{w}}_{h}, \Phi_{h}\right):=\sum_{\Gamma \in \mathcal{F}_{h}^{I}} \int_{\Gamma} \sum_{s=1}^{2} \mathbf{k}_{s}\left(\overline{\mathbf{w}}_{h}^{L}\right) \cdot \Phi_{h}^{L}\left(\boldsymbol{n}_{\Gamma}\right)_{s} \mathrm{~d} S \\
& -\sum_{\Gamma \in \mathcal{F}_{h}^{I}} \int_{\Gamma} \sum_{s=1}^{2} \mathbf{k}_{s}\left(\overline{\mathbf{w}}_{h}^{R}\right) \cdot \Phi_{h}^{R}\left(\boldsymbol{n}_{\Gamma}\right)_{s} \mathrm{~d} S \\
& +\sum_{\Gamma \in \mathcal{F}_{h}^{B}} \int_{\Gamma} \sum_{s=1}^{2} \mathbf{k}_{s}\left(\overline{\mathbf{w}}_{h}\right) \cdot \Phi_{h}\left(\boldsymbol{n}_{\Gamma}\right)_{s} \mathrm{~d} S \\
& -\sum_{K \in \mathcal{T}_{h}} \int_{K} \sum_{s=1}^{2} \mathbf{k}_{s}\left(\overline{\mathbf{w}}_{h}\right) \cdot \frac{\partial \Phi_{h}}{\partial x_{s}} \mathrm{~d} \boldsymbol{x}, \\
& J_{h}^{\sigma}\left(\mathbf{w}_{h}, \Phi_{h}\right):=\sum_{\Gamma \in \mathcal{F}_{h}^{I}} \int_{\Gamma} \sigma\left[\mathbf{w}_{h}\right] \cdot\left[\Phi_{h}\right] \mathrm{d} S \\
& +\sum_{\Gamma \in \mathcal{F}_{h}^{B}} \int_{\Gamma} \sigma \mathbf{w}_{h} \cdot \Phi_{h} \mathrm{~d} S, \\
& l_{h}\left(\overline{\mathbf{w}}_{h}, \Phi_{h}\right):=\sum_{\Gamma \in \mathcal{F}_{h}^{B}} \int_{\Gamma} \sigma \mathbf{w}_{B} \cdot \Phi_{h} \mathrm{~d} S \\
& -\Theta \sum_{\Gamma \in \mathcal{F}_{h}^{B}} \int_{\Gamma} \sum_{s=1}^{2} \sum_{k=1}^{2} \mathbf{K}_{k, s}^{T}\left(\overline{\mathbf{w}}_{h}\right) \frac{\partial \Phi_{h}}{\partial x_{k}}\left(\boldsymbol{n}_{\Gamma}\right)_{s} \cdot \mathbf{w}_{B} \mathrm{~d} S,
\end{aligned}
$$

where $\sigma$ is a parameter of the method and boundary state $\mathbf{w}_{B}$ is defined on the basis of the Dirichlet boundary conditions and extrapolation.

In the vicinity of discontinuities or steep gradients nonphysical oscillations can appear in the approximate solution. In order to overcome this difficulty we employ artificial viscosity forms, see [3]. They are based on the discontinuity indicator

$$
g(K):=\frac{1}{d(K)|K|^{3 / 4}} \int_{\partial K}\left[\bar{\rho}_{h}\right]^{2} d S, \quad K \in \mathcal{T}_{h},
$$

where $\left[\bar{\rho}_{h}\right]$ is the jump of the function $\bar{\rho}_{h}$ (= the first component of the vector function $\overline{\mathbf{w}}_{h}$ ) on the boundary $\partial K$, $d(K)$ denotes the diameter of $K$ and $|K|$ denotes the area of the element $K$. Then we define the discrete discontinuity indicator

$$
\begin{array}{ll}
G(K):=0 & \text { if } g(K)<1, \\
G(K):=1 & \text { if } g(K) \geq 1, K \in \mathcal{T}_{h},
\end{array}
$$

and the artificial viscosity forms

$$
\begin{aligned}
& \tilde{\beta}_{h}\left(\overline{\mathbf{w}}_{h}, \mathbf{w}_{h}, \Phi_{h}\right):= \\
& v_{1} \sum_{K \in \mathcal{T}_{h}} d(K) G(K) \int_{K} \nabla \mathbf{w}_{h} \cdot \nabla \Phi_{h} \mathrm{~d} \boldsymbol{x},
\end{aligned}
$$

$$
\begin{aligned}
& \tilde{J}_{h}\left(\overline{\mathbf{w}}_{h}, \mathbf{w}_{h}, \Phi_{h}\right):= \\
& v_{2} \sum_{\Gamma \in \mathcal{F}_{h}^{\prime}} \frac{1}{2}\left(G\left(K_{\Gamma}^{L}\right)+G\left(K_{\Gamma}^{R}\right)\right) \int_{\Gamma}\left[\mathbf{w}_{h}\right] \cdot\left[\Phi_{h}\right] \mathrm{d} S,
\end{aligned}
$$

with constants $v_{1}$ and $v_{2}$.

All these forms are linear with respect to $\mathbf{w}_{h}$ and nonlinear with respect to $\overline{\mathbf{w}}_{h}$.

Finally, we set

$$
(\varphi, \psi)=\int_{\Omega_{h}} \varphi \psi \mathrm{d} x .
$$

\subsection{Full space-time DG discretization}

Let $0=t_{0}<t_{1}<\ldots<t_{M}=T$ be a partition of the interval $[0, T]$ and let us denote $I_{m}=\left(t_{m-1}, t_{m}\right), \tau_{m}=t_{m}-t_{m-1}$ for $m=1, \ldots, M$. We define the space $\mathbf{S}_{h, \tau}^{p, q}=\left(S_{h, \tau}^{p, q}\right)^{4}$, where

$$
S_{h, \tau}^{p, q}:=\left\{\phi ;\left.\phi\right|_{I_{m}}=\sum_{i=0}^{q} \zeta_{i} \phi_{i} \text {, where } \phi_{i} \in S_{h}^{p}, \zeta_{i} \in P^{q}\left(I_{m}\right)\right\} .
$$

with integers $p, q \geq 1$. $P^{q}\left(I_{m}\right)$ denotes the space of all polynomials in $t$ on $I_{m}$ of degree $\leq q$. Moreover for $\Phi \in \mathbf{S}_{h, \tau}^{p, q}$ we introduce the following notation:

$$
\begin{aligned}
& \Phi_{m}^{ \pm}=\Phi\left(t_{m}^{ \pm}\right)=\lim _{t \rightarrow t_{m \pm}} \Phi(t), \\
& \{\Phi\}_{m}=\Phi_{m}^{+}-\Phi_{m}^{-} .
\end{aligned}
$$

Approximate solution $\mathbf{w}_{h \tau}$ of the problem will be sought in the space $\mathbf{S}_{h, \tau}^{p, q}$. Since the functions of this space are in general discontinuous in time, we ensure the connection between $I_{m-1}$ and $I_{m}$ by the penalty term in time

$$
\left(\left\{\mathbf{w}_{h \tau}\right\}_{m-1}, \Phi_{h \tau}\left(t_{m-1}^{+}\right)\right) .
$$

The initial state $\mathbf{w}_{h \tau}$ is included by the $L^{2}\left(\Omega_{h}\left(t_{0}\right)\right)$-projection of $\mathbf{w}^{0}$ on $\mathbf{S}_{h}^{p}\left(t_{0}\right)$ :

$$
\left(\mathbf{w}_{h \tau}\left(t_{0}^{+}\right), \Phi_{h \tau}\left(t_{0}^{+}\right)\right)=\left(\mathbf{w}^{0}, \Phi_{h \tau}\left(t_{0}^{+}\right)\right) \quad \forall \Phi_{h \tau} \in \mathbf{S}_{h, \tau}^{p, q} .
$$

Now we introduce a suitable linearization. We can use two possibilities.

1) We put $\overline{\mathbf{w}}_{h \tau}(t):=\mathbf{w}_{h}\left(t_{m-1}^{-}\right)$for $t \in I_{m}$.

2) We prolong the solution from the time interval $I_{m-1}$ to the time interval $I_{m}$.

We say that a function $\mathbf{w}_{h \tau} \in \mathbf{S}_{h, \tau}^{p, q}$ is the approximate solution of the problem (1) obtained by the ST-DG method, if it satisfies the following conditions

$$
\begin{aligned}
& \sum_{m=1}^{M} \int_{I_{m}}\left(\frac{\partial \mathbf{w}_{h \tau}}{\partial t}, \Phi_{h \tau}\right) \mathrm{d} t \\
& +\sum_{m=1}^{M} \int_{I_{m}}\left(a_{h}\left(\overline{\mathbf{w}}_{h \tau}, \mathbf{w}_{h \tau}, \Phi_{h \tau}\right)+b_{h}\left(\overline{\mathbf{w}}_{h \tau}, \mathbf{w}_{h \tau}, \Phi_{h \tau}\right)\right) \mathrm{d} t \\
& +\sum_{m=1}^{M} \int_{I_{m}}\left(\tilde{\beta}_{h}\left(\overline{\mathbf{w}}_{h \tau}, \mathbf{w}_{h \tau}, \Phi_{h \tau}\right)+\tilde{J}_{h}\left(\overline{\mathbf{w}}_{h \tau}, \mathbf{w}_{h \tau}, \Phi_{h \tau}\right)\right) \mathrm{d} t \\
& +\sum_{m=1}^{M} \int_{I_{m}}\left(J_{h}^{\sigma}\left(\mathbf{w}_{h \tau}, \Phi_{h \tau}\right)+p_{h}\left(\overline{\mathbf{w}}_{h \tau}, \mathbf{w}_{h \tau}, \Phi_{h \tau}\right)\right) \mathrm{d} t
\end{aligned}
$$




$$
\begin{aligned}
& +\sum_{m=2}^{M}\left(\left\{\mathbf{w}_{h \tau}\right\}_{m-1}, \Phi_{h \tau}\left(t_{m-1}^{+}\right)\right)+\left(\mathbf{w}_{h \tau}\left(t_{0}^{+}\right), \Phi_{h \tau}\left(t_{0}^{+}\right)\right) \\
& =\sum_{m=1}^{M} \int_{I_{m}} l_{h}\left(\overline{\mathbf{w}}_{h \tau}, \Phi_{h \tau}\right) \mathrm{d} t+\left(\mathbf{w}^{0}, \Phi_{h \tau}\left(t_{0}^{+}\right)\right) \\
& +\sum_{m=1}^{M} \int_{I_{m}} k_{h}\left(\overline{\mathbf{w}}_{h \tau}, \Phi_{h \tau}\right) \mathrm{d} t \quad \forall \Phi_{h \tau} \in \mathbf{S}_{h, \tau}^{p, q} .
\end{aligned}
$$

\section{Discretization of the $k-\omega$ turbulence model}

\subsection{Space discretization of the problem}

We apply discontinuous Galerkin method in the similar way as in the previous section. Discretization is carried out on the same mesh for simplicity. An approximate solution of the problem is looked for in the space $\tilde{\mathbf{S}}_{h}^{\tilde{p}}=\left(S_{h}^{\tilde{p}}\right)^{2}$ and a function $\tilde{\Phi} \in \tilde{\mathbf{S}}_{h}^{\tilde{p}}$ is, in general, discontinuous on interfaces $\Gamma \in \mathcal{F}_{h}^{I}$.

If we define $\tilde{\mathbf{P}}^{ \pm}=\left(\langle\rho \mathbf{v}\rangle \cdot \boldsymbol{n}_{\Gamma}\right)^{ \pm} \mathbf{I}$, where $\mathbf{I}=\operatorname{diag}(1,1)$, and use the property (5) then we can define convect form

$$
\begin{aligned}
& \tilde{b}_{h}\left(\overline{\tilde{\mathbf{w}}}_{h}, \tilde{\mathbf{w}}_{h}, \tilde{\Phi}_{h}\right):= \\
& -\sum_{K \in \mathcal{T}_{h}} \int_{K} \sum_{s=1}^{2} \tilde{\mathbf{A}}_{s} \tilde{\mathbf{w}}_{h} \cdot \frac{\partial \tilde{\Phi}_{h}}{\partial x_{s}} \mathrm{~d} \boldsymbol{x} \\
& +\sum_{\Gamma \in \mathcal{F}_{h}^{I}} \int_{\Gamma}\left(\tilde{\mathbf{P}}^{+} \tilde{\mathbf{w}}_{h}^{L}+\tilde{\mathbf{P}}^{-} \tilde{\mathbf{w}}_{h}^{R}\right) \cdot\left[\tilde{\Phi}_{h}\right] \mathrm{d} S \\
& +\sum_{\Gamma \in \mathcal{F}_{h}^{B}} \int_{\Gamma}\left(\tilde{\mathbf{P}}^{+} \tilde{\mathbf{w}}_{h}^{L}+\tilde{\mathbf{P}}^{-} \tilde{\tilde{\mathbf{w}}}_{h}^{R}\right) \cdot \tilde{\Phi}_{h} \mathrm{~d} S,
\end{aligned}
$$

where the state $\overline{\tilde{\mathbf{w}}}_{h}^{R}$ is based on the boundary conditions.

For the discretization of the diffusion term we use the property (6) and then we have

$$
\begin{aligned}
& \tilde{a}_{h}\left(\overline{\tilde{\mathbf{w}}}_{h}, \tilde{\mathbf{w}}_{h}, \tilde{\Phi}_{h}\right):= \\
& +\sum_{K \in \mathcal{T}_{h}} \int_{K} \sum_{s=1}^{2} \tilde{\mathbf{K}}_{s}\left(\overline{\tilde{\mathbf{w}}}_{h}\right) \frac{\partial \tilde{\mathbf{w}}_{h}}{\partial x_{s}} \cdot \frac{\partial \tilde{\Phi}_{h}}{\partial x_{s}} \mathrm{~d} \boldsymbol{x} \\
& -\sum_{\Gamma \in \mathcal{F}_{h}^{I}} \int_{\Gamma} \sum_{s=1}^{2}\left\langle\tilde{\mathbf{K}}_{s}\left(\tilde{\tilde{\mathbf{w}}}_{h}\right) \frac{\partial \tilde{\mathbf{w}}_{h}}{\partial x_{s}}\right\rangle\left(\boldsymbol{n}_{\Gamma}\right)_{s} \cdot\left[\tilde{\Phi}_{h}\right] \mathrm{d} S \\
& -\sum_{\Gamma \in \mathcal{F}_{h}^{B}} \int_{\Gamma} \sum_{s=1}^{2} \tilde{\mathbf{K}}_{s}\left(\overline{\tilde{\mathbf{w}}}_{h}\right) \frac{\partial \tilde{\mathbf{w}}_{h}}{\partial x_{s}}\left(\boldsymbol{n}_{\Gamma}\right)_{s} \cdot \tilde{\Phi}_{h} \mathrm{~d} S \\
& -\Theta \sum_{\Gamma \in \mathcal{F}_{h}^{I}} \int_{\Gamma} \sum_{s=1}^{2}\left\langle\tilde{\mathbf{K}}_{s}\left(\tilde{\tilde{\mathbf{w}}}_{h}\right) \frac{\partial \tilde{\Phi}_{h}}{\partial x_{s}}\right\rangle\left(\boldsymbol{n}_{\Gamma}\right)_{s} \cdot\left[\tilde{\mathbf{w}}_{h}\right] \mathrm{d} S \\
& -\Theta \sum_{\Gamma \in \mathcal{F}_{h}^{B}} \int_{\Gamma} \sum_{s=1}^{2} \tilde{\mathbf{K}}_{s}\left(\overline{\tilde{\mathbf{w}}}_{h}\right) \frac{\partial \tilde{\Phi}_{h}}{\partial x_{s}}\left(\boldsymbol{n}_{\Gamma}\right)_{s} \cdot \tilde{\mathbf{w}}_{h} \mathrm{~d} S .
\end{aligned}
$$

Further we define the turbulent form $\tilde{s}_{h}$, the interior and boundary penalty form $\tilde{J}_{h}^{\tilde{\sigma}}$ and the right-hand side form $\tilde{l}_{h}$ in the following way:

$$
\tilde{J}_{h}^{\tilde{\sigma}}\left(\tilde{\mathbf{w}}_{h}, \tilde{\Phi}_{h}\right):=\sum_{\Gamma \in \mathcal{F}_{h}^{l}} \int_{\Gamma} \tilde{\sigma}\left[\tilde{\mathbf{w}}_{h}\right] \cdot\left[\tilde{\Phi}_{h}\right] \mathrm{d} S
$$

$$
\begin{aligned}
& +\sum_{\Gamma \in \mathcal{F}_{h}^{B}} \int_{\Gamma} \tilde{\sigma} \tilde{\mathbf{w}}_{h} \cdot \tilde{\Phi}_{h} \mathrm{~d} S, \\
& \tilde{l}_{h}\left(\overline{\tilde{\mathbf{w}}}_{h}, \tilde{\Phi}_{h}\right):=\sum_{\Gamma \in \mathcal{F}_{h}^{B}} \int_{\Gamma} \tilde{\sigma} \tilde{\mathbf{w}}_{B} \cdot \tilde{\Phi}_{h} \mathrm{~d} S \\
& -\Theta \sum_{\Gamma \in \mathcal{F}_{h}^{B}} \int_{\Gamma} \sum_{s=1}^{2} \tilde{\mathbf{K}}_{s}\left(\overline{\tilde{\mathbf{w}}}_{h}\right) \frac{\partial \tilde{\Phi}_{h}}{\partial x_{s}}\left(\boldsymbol{n}_{\Gamma}\right)_{s} \cdot \tilde{\mathbf{w}}_{B} \mathrm{~d} S . \\
& \tilde{\boldsymbol{s}}_{h}\left(\overline{\tilde{\mathbf{w}}}_{h}, \tilde{\Phi}_{h}\right):=\sum_{K \in \mathcal{T}_{h}} \int_{K} \tilde{s}\left(\overline{\tilde{\mathbf{w}}}_{h}\right) \cdot \tilde{\Phi}_{h} \mathrm{~d} \boldsymbol{x}
\end{aligned}
$$

Boundary state $\tilde{\mathbf{w}}_{B}$ is defined on the basis of the Dirichlet boundary conditions and extrapolation.

\subsection{Full space-time DG discretization}

For the full space-time discontinuous Galerkin discretization we use the same partition of the interval $[0, T]$ as in the previous section. We say that a function $\tilde{\mathbf{w}}_{h \tau} \in \tilde{\mathbf{S}}_{h, \tau}^{\tilde{p}, \tilde{q}}$ is the approximate solution of the problem (4) obtained by the ST-DG method, if it satisfies the following conditions

$$
\begin{aligned}
& \sum_{m=0}^{M-1} \int_{I_{m}}\left(\frac{\partial \rho \tilde{\mathbf{w}}_{h \tau}}{\partial t}, \tilde{\Phi}_{h \tau}\right) \mathrm{d} t \\
& +\sum_{m=0}^{M-1} \int_{I_{m}}\left(\tilde{a}_{h}\left(\overline{\tilde{\mathbf{w}}}_{h \tau}, \tilde{\mathbf{w}}_{h \tau}, \tilde{\Phi}_{h \tau}\right)+\tilde{b}_{h}\left(\overline{\tilde{\mathbf{w}}}_{h \tau}, \tilde{\mathbf{w}}_{h \tau}, \tilde{\Phi}_{h \tau}\right)\right) \mathrm{d} t \\
& +\sum_{m=0}^{M-1} \int_{I_{m}} \tilde{J}_{h}^{\tilde{\sigma}}\left(\tilde{\mathbf{w}}_{h \tau}, \tilde{\Phi}_{h \tau}\right) \mathrm{d} t \\
& +\sum_{m=1}^{M-1}\left(\left\{\tilde{\mathbf{w}}_{h \tau}\right\}_{m}, \tilde{\Phi}_{h \tau}\left(t_{m}^{+}\right)\right)+\left(\tilde{\mathbf{w}}_{h \tau}\left(t_{0}^{+}\right), \tilde{\Phi}_{h \tau}\left(t_{0}^{+}\right)\right) \\
& =\sum_{m=0}^{M-1} \int_{I_{m}}\left(\tilde{l}_{h}\left(\overline{\tilde{\mathbf{w}}}_{h \tau}, \tilde{\Phi}_{h \tau}\right)+\tilde{s}_{h}\left(\overline{\tilde{\mathbf{w}}}_{h \tau}, \tilde{\Phi}_{h \tau}\right)\right) \mathrm{d} t \\
& +\left(\tilde{\mathbf{w}}^{0}, \tilde{\Phi}_{h \tau}\left(t_{0}^{+}\right)\right) \quad \forall \tilde{\Phi}_{h \tau} \in \tilde{\mathbf{S}}_{h, \tau}^{\tilde{p}, \tilde{q}} .
\end{aligned}
$$

\section{Numerical experiments}

In order to demonstrate the applicability of the developed method we shall present two numerical simulations of compressible turbulent flow in 2D. For this purpose we chose two regimes for the profile RAE2822 and compared it with experimental data, which can be found in [12]. First regime is characterized by the far-field Mach number $M_{\infty}=0.6$ and the angle of the attack $\alpha=2.57^{\circ}$ ('case 3' in [12]) and second case is characterized by the Mach number $M_{\infty}=$ 0.73 and the angle of the attack $\alpha=3.19^{\circ}$ ('case 9' in [12]). For both cases we set free-stream turbulence intensity $T u=1 \%$. Figures $1-3$ and 5-7 show the pressure distribution, Mach number distribution and turbulent kinetic energy distribution. Comparison of the computed pressure coefficient $c_{p}$ with the experimental data is shown in Figures 4 and 8 . Figure 9 shows detail of the mesh which consist of 64150 elements in total. 


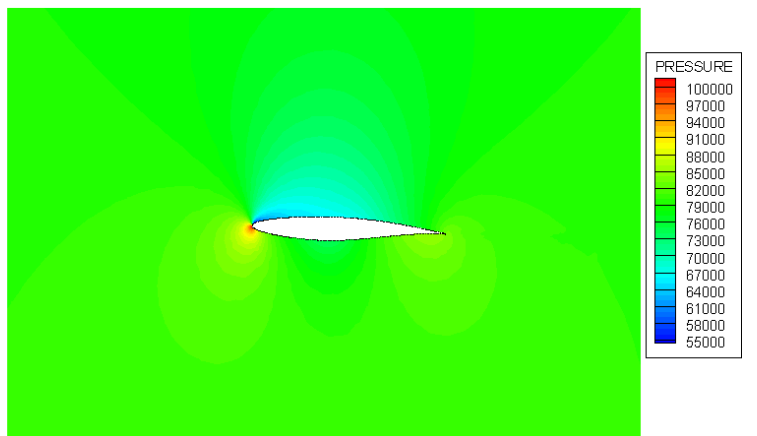

Fig. 1. The distribution of the pressure for the case 3 .

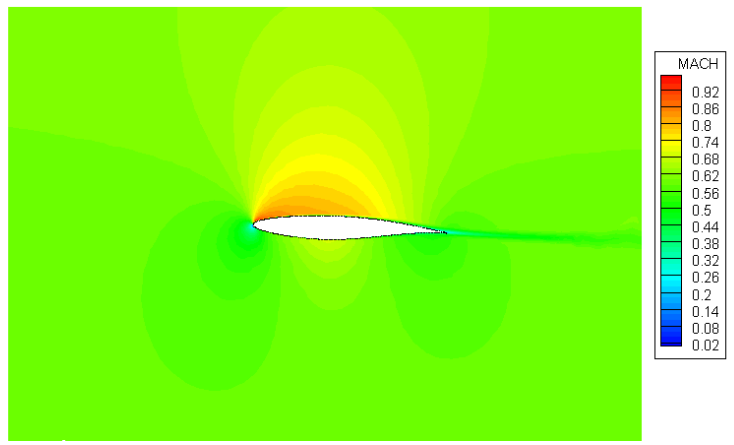

Fig. 2. The distribution of the Mach number for the case 3.

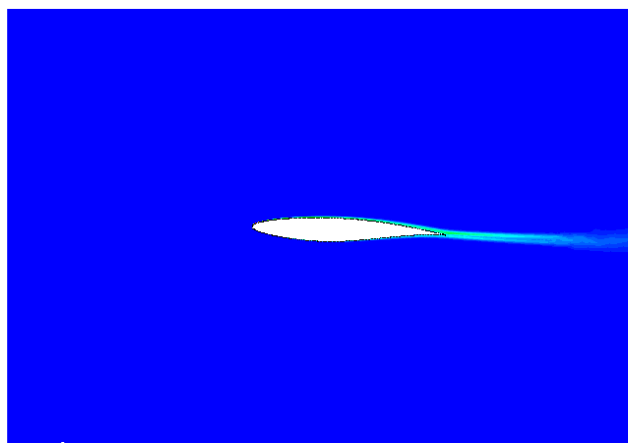

Fig. 3. The distribution of the turbulent kinetic energy for the case 3 .

\section{Conclusion}

In this paper we dealt with the space-time discontinuous Galerkin method for the numerical solution of the viscous compressible turbulent flow. The applicability of the proposed method was demonstrated on the examples which show that the presented method is an efficient numerical scheme for the solution of the viscous compressible turbulent flow.

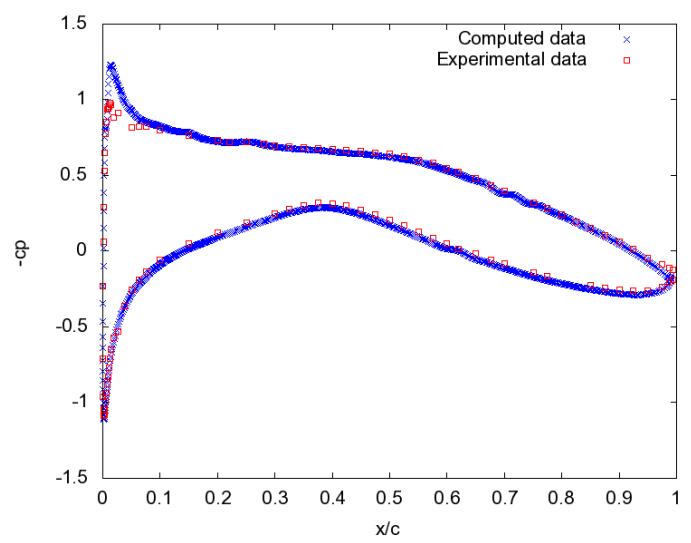

Fig. 4. Comparison of the computed pressure coefficient $c_{p}$ with the experimental data for the case 3 .

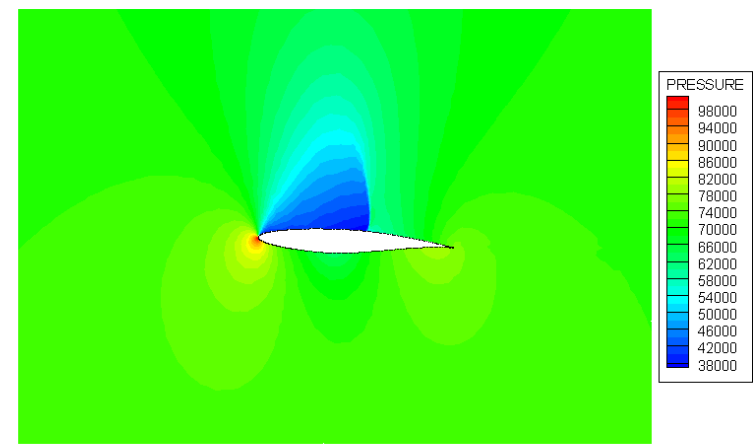

Fig. 5. The distribution of the pressure for the case 9 .

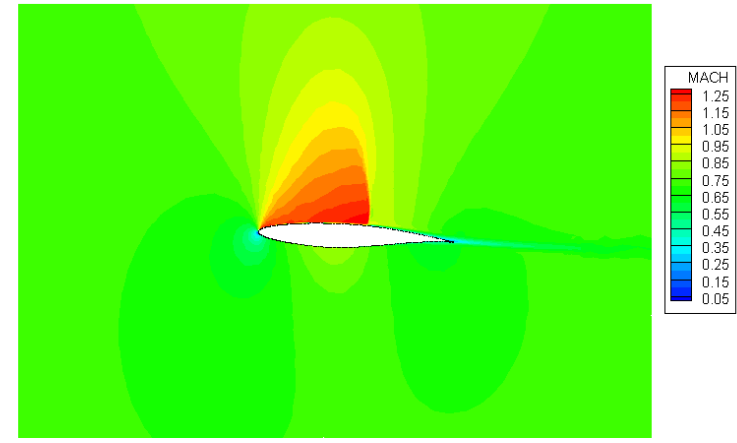

Fig. 6. The distribution of the Mach number for the case 9 .

\section{Acknowledgment}

This result originated with the support of Ministry of Industry and Trade of the Czech Republic for the long-term strategic development of the research organization. The author acknowledge this support. 

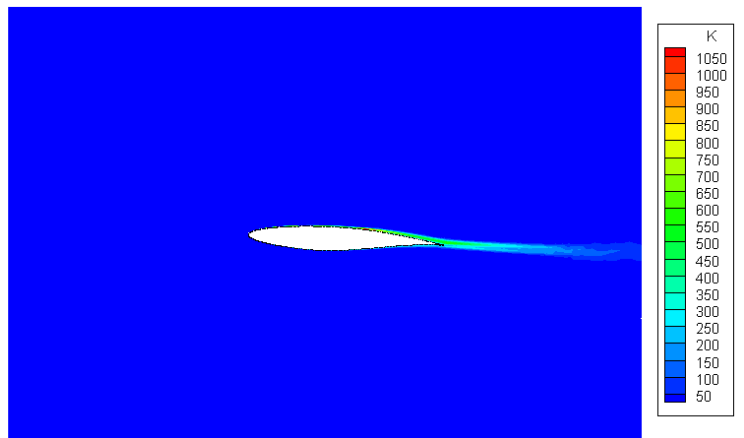

Fig. 7. The distribution of the turbulent kinetic energy for the case 9 .

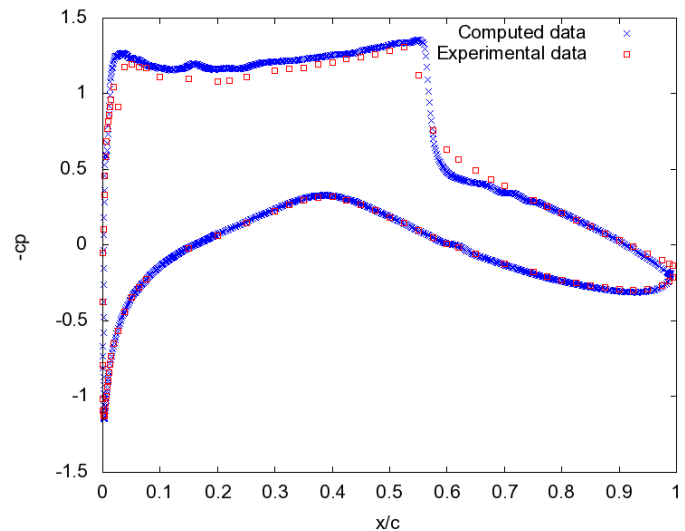

Fig. 8. Comparison of the computed pressure coefficient $c_{p}$ with the experimental data for the case 9 .

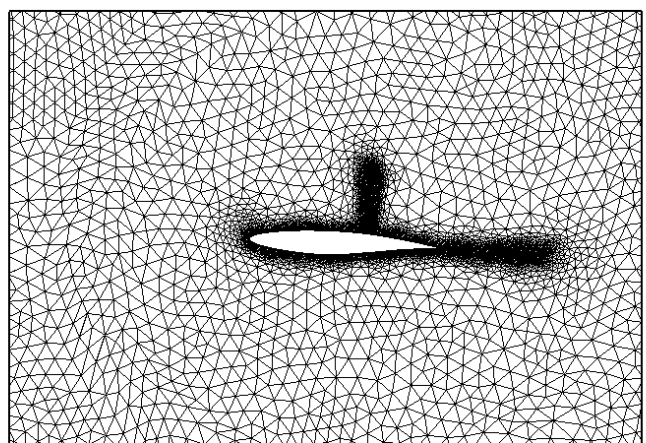

Fig. 9. Detail of the mesh.

\section{References}

1. M. Feistauer, J. Felcman, and I. Straškraba Mathematical and Computational Methods for Compressible Flow, Clarendon Press, Oxford (2003)

2. J. Česenek, Discontinuous Galerkin method for solving compressible viscous flow, Doctoral Thesis, (2011)

3. V. Dolejší, M. Feistauer, Discontinuous Galerkin Method, Springer (2015)

4. J. Česenek, et al., Appl. Math. Comput., doi:10.1016/1.amc.2011.08.077 (2011)
5. J. Česenek, M. Feistauer, A. Kosik, ZAMM - Z. Angew. Math. Mech. 93, 6 - 7, 387 - 402 (2013)

6. J. Česenek, EPJ WoC 67, 02015 (2014)

7. J. Česenek, Combined finite volume - space-time discontinuous Galerkin method for the 2D compressible turbulent flow. Technical report R-6154, VZLÚ (2014)

8. J. Česenek, EPJ WoC 114, 02012 (2016)

9. J. Česenek, EPJ WoC 143, 02014 (2017)

10. D.C. Wilcox, AIAA Journal 46,11, (2008)

11. Bassi, et al., Computers and Fluids 34, 507-540 (2005)

12. P.H. Cook, M.A. McDonald and M.C.P. Firmin, AGARD Advisory Report 138, (1979) 